\title{
Do changes in inflammatory markers predict hepatocellular carcinoma recurrence and survival after liver transplantation?
}

\author{
Lucas José Caram ${ }^{1, *}$, Francisco Calderon ${ }^{1, *}$, Esteban Masino ${ }^{1, *}$, Victoria Ardiles ${ }^{1}$, Ezequiel Mauro ${ }^{2}$, Leila Haddad ${ }^{2}$, \\ Juan Pekolj ${ }^{1}$, Jimena Vicens ${ }^{3}$, Adrian Gadano ${ }^{2}$, Eduardo de Santibañes ${ }^{1}$, Martín de Santibañes ${ }^{1}$ \\ 'Division of Hepato Pancreatic Biliary Surgery and Liver Transplant Unit, Department of General Surgery, \\ Hospital Italiano de Buenos Aires, Buenos Aires, Argentina, \\ 2Department of Internal Medicine and Hepatology, Hospital Italiano de Buenos Aires, Buenos Aires, Argentina, \\ ${ }^{3}$ Department of Internal Medicine and Statistics, Hospital Italiano de Buenos Aires, Buenos Aires, Argentina
}

\begin{abstract}
Backgrounds/Aims: The role of inflammation in malignant cell proliferation has been well described. High values of platelet-to-lymphocyte ratio (PLR) and neutrophil-to-lymphocyte ratio (NLR) as markers of systemic inflammation have shown associations with unfavorable long-term outcomes. The purpose of this study was to determine values of NLR and PLR evaluated prior to and after surgery and their associations with mortality and recurrence rates of liver transplant patients with hepatocellular carcinoma (HCC).

Methods: A total of 105 patients with HCC who underwent orthotopic liver transplantation (OLT) were retrospectively reviewed. NLR and PLR values were obtained from complete blood counts prior to and after surgery. Overall survival (OS) and recurrence-free survival (RFS) in relation with delta NLR and PLR were estimated.

Results: Serum alpha-fetoprotein levels $>100 \mathrm{ng} / \mathrm{mL}(p=0.014)$ and lymphovascular emboli in the specimen $(p=0.048)$ were identified to be significant predictors of RFS. Child-Pugh score $(p=0.016)$ was found to be an independent factor associated with poorer OS. An increasing delta PLR was associated with worse RFS, although it showed no significant association with OS.

Conclusions: The analysis of PLR as a continuous variable may predict recurrence outcomes in patients undergoing OLT for HCC. It is more representative than isolated values.
\end{abstract}

Key Words: Transplantation; Liver neoplasms; Recurrence; Neutrophils; Platelets

Received: June 23, 2021, Revised: September 2, 2021, Accepted: September 21, 2021

Corresponding author: Martín de Santibañes Division of Hepato Pancreatic Biliary Surgery and Liver Transplant Unit, Department of General Surgery, Hospital Italiano de Buenos Aires, Juan D. Perón 4190, Buenos Aires, C1181ACH, Argentina

Tel: +54-11-4981-4501, Fax: +54-00-4958-2200,

E-mail: martin.desantibanes@hospitalitaliano.org.ar

ORCID: https://orcid.org/0000-0002-0163-1248

*These authors contributed equally to this study.

\section{INTRODUCTION}

Hepatocellular carcinoma (HCC) is the sixth most common cancer and the fourth leading cancer in mortality worldwide [1]. Each year, approximately 750,000 new cases of HCC are diagnosed [1]. It has been identified as the primary cause of mortality in cirrhotic individuals. Its incidence is predicted to increase in the future [2].

Liver resection (LR), orthotopic liver transplantation (OLT), radiofrequency ablation (RFA), transarterial embolization (TAE)/transarterial chemoembolization (TACE), radiation therapy (RT), systemic therapy, and best supportive care are current therapeutic options for HCC [3]. Currently, only LR, RFA, and OLT are curative therapies for HCC $[1,4,5]$. In theory, OLT is considered the "gold standard" because it can cure both the tumor and the underlying cirrhosis simultaneously [6]. The
\end{abstract}


Milan criteria are the benchmark to offer the best post-OLT survival in HCC (> 70\% of 5-year survival with a recurrence rate $<10 \%-15 \%$ ) [4]. In spite of available therapies, prognosis of HCC after treatment remains poor due to multi-centricity of HCC in a cirrhotic liver with a low disease-free survival (DFS) rate generally. Thus, risk stratification and patient selection for potentially curative resection among other factors are crucial. Multiple factors associated with aggressiveness and progression of HCC, such as tumor number, size, macrovascular invasion, and serum alpha-fetoprotein (AFP) levels, have been reported [7]. Identifying other predictors such as biological serum markers for HCC recurrence and survival remains a subject of active research by the scientific community.

The role of systemic inflammation in malignant cell proliferation and survival has been well described. Inflammatory injury secondary to malignant processes results in the up-regulation of cytokines, causing an increased risk for malignancy by several pathways: suppression of apoptosis, angiogenesis, and direct DNA damage $[8,9]$. Neutrophil-to-lymphocyte ratio (NLR) and platelet-to-lymphocyte ratio (PLR) have gained significant scientific spotlight in the last several years. Multiple studies have suggested that elevated values of NLR and PLR are associated with poor long-term results after a curative-intent surgery for patients with several different oncologic afflictions [10,11].

NLR and PLR have been reported to be independent factors for HCC recurrence and survival $[10,12]$. However, the prognostic role of NLR and PLR obtained at different stages of surgical treatment remains unknown. Cut-off values are yet to be established for both of these variables regarding HCC. Delta NLR/PLR, or the difference between their postoperative and preoperative values, can be expressed as an increasing or decreasing trend. If we consider disease and treatment of patients not as static elements, but rather as dynamic factors which constantly interact with each other, we must take into consideration changes in inflammatory parameters before and after treatment. Delta NLR is correlated with survival and recurrence in other pathologies such as oesophageal cancer [13].

Thus, the purpose of the present study was to determine the prognostic usefulness of NLR and PLR, their trend (delta NLR/ PLR) measured at two points (before and after surgery), and their relationship with mortality and recurrence rates in liver transplant patients with HCC.

\section{PATIENTS AND METHODS}

\section{Population}

This retrospective, single-center study included all adult (> 18-year-old) patients registered to our hospital's healthcare system who underwent OLT for HCC between 2007 and 2018. Information was gathered from the institutional electronic health records. Imaging/procedure reports, admission notes, intraoperative and postoperative findings, and discharge summaries were input into a retrospective electronic database. This study's conduct was approved by the Institutional Ethics Committee for Investigative Protocols (no. 3474).

Primary end-points were overall survival (OS) and recurrence-free survival (RFS) in relation with delta NLR and PLR. Secondary end-points were variables associated with mortality and recurrence.

Baseline characteristics such as patient sex, age, liver fibrosis, and cirrhosis etiology were obtained. Child-Pugh score and Barcelona Clinic Liver Cancer classification (BCLC) at the moment of diagnosis were recorded. "Bridging" therapies such as TACE and transarterial radioembolization (TARE) were also recorded. Vascular invasion, Edmondson-Steiner grade, number of tumors, and the largest tumor were determined by reviewing pathology reports.

Staging was performed using a combination of blood tests, ultrasonography (fibroscan), computed tomography, positron emission tomography scans, and magnetic resonance imaging according to the BCLC based on tumor size, underlying liver disease, and patient's functional status. LR, RFA, and OLT were considered primary treatment methods [5,14]. Alternative therapies included TAE/TACE and multiple tyrosine kinase inhibitors such as sorafenib when curative treatment was not a feasible option [15].

All patients were enrolled in the transplant wait list and assigned points via Milan exception rules according to Argentinian transplantation laws. Patients received OLT in accordance to Milan criteria. After discharge, patients were regularly followed-up by a multidisciplinary team, including attending surgeons and hepatologists. Follow-up visits were performed weekly in the first two months and monthly thereafter.

Recurrence was defined as clinical or radiological evidence of tumoral relapse after adequate treatment following a disease-free period of time. The date of recurrence was defined as the date on which the recurrence was confirmed based on radiological findings. The DFS period was calculated from the day of the OLT to the date of the first recurrence or until the loss of follow-up. OS was defined as the time between OLT and subject death of any cause.

\section{Laboratory data}

AFP values closest to the OLT were obtained. NLR and PLR values were obtained preoperatively within one month prior to surgery and postoperatively at approximately 90 days after surgery. All complete blood counts (CBC) obtained within the specified period were assessed. NLR and PLR were calculated for each CBC by dividing the absolute neutrophil and platelet value by the absolute lymphocyte count, respectively. Their highest values within the specified time frame were used for analysis. Delta NLR or PLR was calculated as the difference between postoperative NLR or PLR and preoperative NLR or PLR. Delta NLR and delta PLR were expressed as numerical values as well as categorical variables with "increasing" $(>0)$ or "decreasing" $(<0)$ trends. 


\section{Exclusion criteria}

Patients $<18$ years were excluded. Patients who underwent "curative treatments" before OLT (LR and/or RFA), patients with a prior OLT, and patients with an anatomical and pathological findings of mixed "hepatocholangiocarcinoma" were also excluded. OLT patients with HCC that lacked proper preoperative and/or postoperative follow-up and lab results were also excluded.

\section{Statistical analysis}

Data are presented as either mean and standard deviation or median and interquartile ranges for continuous variables and proportions in the case of discrete variables as appropriate. To compare survival between delta NLR/PLR groups, KaplanMeier plots and log-rank tests were employed. Multivariate Cox regression analysis was performed to identify independent predictors of OS and RFS.

A $p$-value of less than 0.05 was considered statistically significant. All analyses were performed using Stata 14 software (StataCorp, College Station, TX, USA). Missing values constituted less than $10 \%$ of the study sample.

\section{RESULTS}

\section{Baseline characteristics}

Of 105 OLT with HCC in the database, only 94 pathological specimens met our inclusion and exclusion criteria (Fig. 1). Two patients were excluded for being under 18 years, one was excluded on the basis of anatomical and pathological findings, and another subject was excluded because of a prior OLT. Seven were omitted due to insufficient information in their medical records. Baseline characteristics of patients analyzed in this study are shown in Table 1. Of 94 subjects selected for this study, the mean age was 58 years at the time of diagnosis, 72 patients (76.6\%) were male. A total of 92 (97.9\%) patients

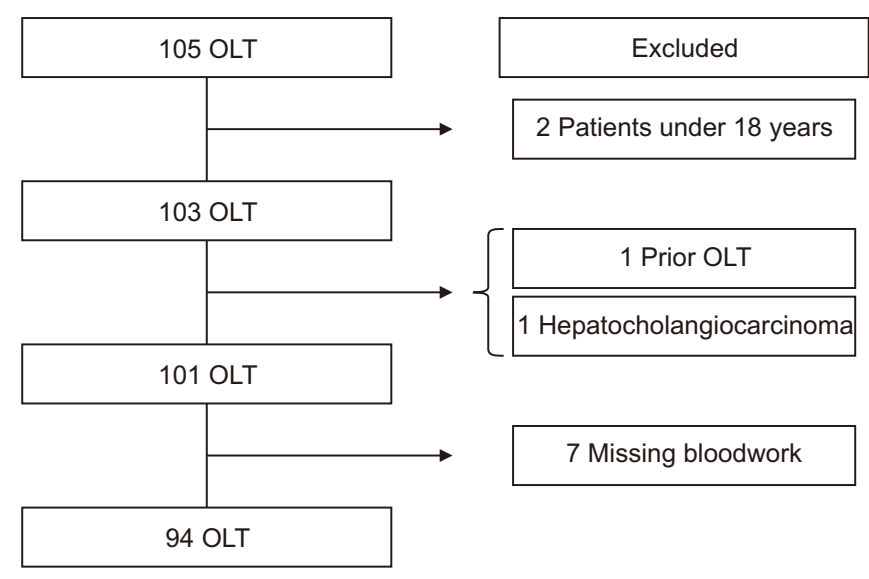

Fig. 1. Selection of patients included in this study. OLT, orthotopic liver transplantation. had cirrhosis. The majority (71.3\%) of them were transplanted with a BCLC staging A, 17.0\% with a BCLC 0 (16 patients), and $11.7 \%$ with a BCLC B. A total of 51 (54.3\%) patients underwent bridging therapy (either TAE, TARE, TACE, or alcoholization). Regarding specimen analysis, most samples corresponded to Edmondson-Steiner grades II and III $(45.7 \%$ and $33.0 \%$, respectively). Thirty-seven (39.4\%) patients showed lymphovascular invasion/emboli. NLR, PLR, delta NLR/PLR, and their trends are presented in Table 2. Delta NLR was 0.30, with an increasing trend in 41 patients. Median delta PLR was 3,282.43, with 70 (74.5\%) patients having an increasing trend.

OS for these patients at 1,3 , and 5 years were $86.3 \%, 75.3 \%$ and $70.1 \%$, respectively. Mean follow-up time was 45.16 months. RFS rates at 1,3 , and 5 years were $97.5 \%, 88.4 \%$, and $82.2 \%$, respectively. OS and RFS Kaplan-Meier graphs are shown in Fig. 2 and 3, respectively.

Table 1. Patient and procedure characteristics (total $=94$ )

\begin{tabular}{|c|c|}
\hline Characteristic & Value \\
\hline Sex (male) & $72(76.6)$ \\
\hline Age (yr) & $58.37 \pm 11.66$ \\
\hline Neoadjuvant chemotherapy & $51(54.3)$ \\
\hline \multicolumn{2}{|l|}{ Edmondson-Steiner } \\
\hline 1 & $5(5.3)$ \\
\hline II & $43(45.7)$ \\
\hline III & $31(33.0)$ \\
\hline IV & $15(16.0)$ \\
\hline Largest nodule (mm) & $29.59 \pm 23.28$ \\
\hline Tumor size (mm) & $36.48 \pm 23.70$ \\
\hline \multicolumn{2}{|l|}{ Tumor number } \\
\hline 1 & $49(52.1)$ \\
\hline $2-3$ & $31(33.0)$ \\
\hline More than 3 & $14(14.9)$ \\
\hline Cirrhosis & $92(97.9)$ \\
\hline \multicolumn{2}{|l|}{$\mathrm{BCLC}$} \\
\hline 0 & $16(17.0)$ \\
\hline$A$ & $67(71.3)$ \\
\hline $\mathrm{B}$ & $11(11.7)$ \\
\hline $\mathrm{C}$ & $0(0)$ \\
\hline $\mathrm{D}$ & $0(0)$ \\
\hline \multicolumn{2}{|l|}{ Child-Pugh } \\
\hline A & $33(35.1)$ \\
\hline B & $33(35.1)$ \\
\hline C & $28(29.8)$ \\
\hline \multicolumn{2}{|l|}{ AFP } \\
\hline$<400$ & $78(83.0)$ \\
\hline$>400$ & $10(10.6)$ \\
\hline Lymphovascular invasion & $37(39.4)$ \\
\hline Follow-up time (mon) & $45.16(17.33-62.6)$ \\
\hline
\end{tabular}

Values are presented as number (\%), mean \pm standard deviation, or median (interquartile range).

BCLC, Barcelona Clinic Liver Cancer classification; AFP, alpha-fetoprotein. 
Table 2. NLR and PLR ratios and deltas in orthotopic liver transplantation patients

\begin{tabular}{lc}
\hline \multicolumn{1}{c}{ Variable } & Value \\
\hline Preoperative NLR & $4.04(0.58-58.82)$ \\
Postoperative NLR & $4.34(0.58-48.65)$ \\
Delta NLR & $0.30(-54.82-31.50)$ \\
Increasing trend & $41(43.6)$ \\
Preoperative PLR & $5,845.82(972.25-120,759.5)$ \\
Postoperative PLR & $9,214.333(301.6453-41,325.72)$ \\
Delta PLR & $3,282.43(-114,116-38,377.55)$ \\
Increasing trend & $70(74.5)$ \\
\hline
\end{tabular}

Values are presented as median (interquartile range) or number (\%).

NLR, neutrophil-to-lymphocyte ratio; PLR, platelet-to-lymphocyte ratio.

\section{Cox regression analysis for overall survival and recurrence-free survival}

Univariate analysis using Cox regression was performed for several variables. Results are presented in Table 3. A higher Child-Pugh score $(p=0.023)$ and a greater total tumor size (sum of maximum diameters of nodules in imaging studies) ( $p$ $=0.05)$ were identified as relevant factors for OS. AFP levels $>$ $100 \mathrm{ng} / \mathrm{mL}(p=0.014)$, lymphovascular invasion/emboli $(p=$ $0.048)$, and an ascending/positive delta PLR trend $(p=0.032)$ were associated with poorer RFS. An increasing trend in both delta NLR and PLR failed to show statistical significance ( $p=$ 0.069 and $p=0.302$, respectively) in OS. These variables were also assessed for their association with RFS rates.

Multivariate analysis was carried out for both OS and RFS. Only a higher Child-Pugh score $(p=0.016)$ was found to be an independent factor associated with poorer OS. AFP levels $>100 \mathrm{ng} / \mathrm{mL}$ (HR, 3.91; 95\% CI, 1.18-12.95; $p=0.026$ ) and an ascending delta PLR (HR, 3.46; 95\% CI, 1.04-11.49; $p=0.042$ ) were independent predictors of RFS. These results are also pre-

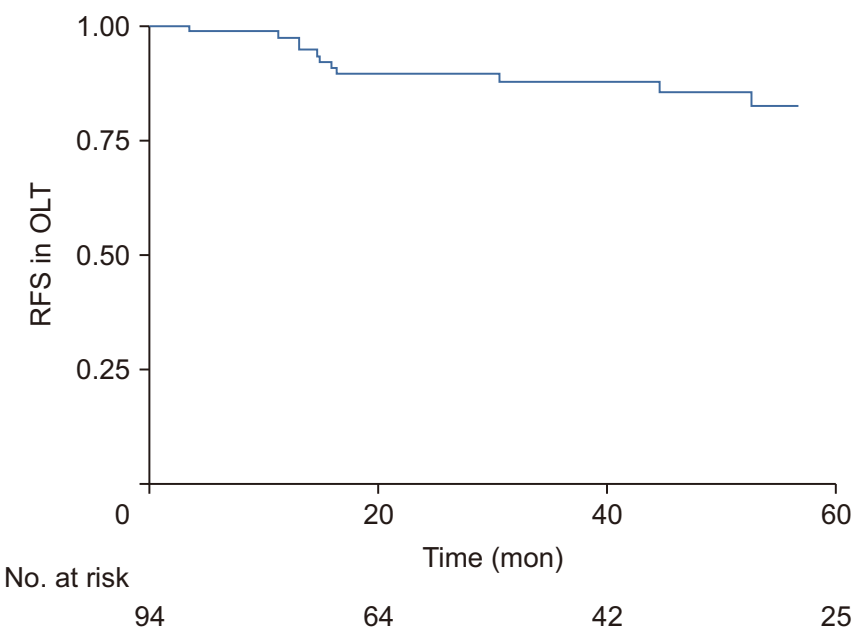

Fig. 2. The Kaplan-Meier curve showing recurrence-free survival (RFS) of orthotopic liver transplantation (OLT) patients. sented in Table 3. Delta NLR/ PLR was used in lieu of pre- and post-operative PLR in the regression analysis because the same values were used to calculate delta PLR. Inclusion of these values would make the regression redundant and decrease its power with little or no benefit.

\section{Relationship of PLR and NLR trends with overall survival and recurrence-free survival}

Patients were divided into two groups according to changes in NLR and PLR. Category 1 consisted of those with negative delta values, whereas category 2 consisted of those with positive delta values. Increasing delta PLR was associated with worse RFS, although it showed no significant association with OS. Delta NLR failed to show significant association with RFS or OS. RFS rates with ascending and descending trends at 1,3 , and 5 years were $98.5 \%, 91.1 \%, 91.1 \%$, and $94.3 \%, 78.4 \%, 57.2 \%$, respectively, with a $\log$ rank test value of $p=0.022$. These results are shown in Fig. 4.

\section{DISCUSSION}

Systemic inflammation is a well-established physiopathological factor in any neoplastic conditions. NLR and PLR are both readily available markers of systemic inflammation. Neutrophilia and hyperplaquetosis reflect an underlying neoplastic inflammatory syndrome. They are usually accompanied by a variable degree of lymphopenia. The balance between negative effects of neutrophilia/platelets and positive effects of lymphocyte-mediated immunity is clearly depicted by these ratios [16]. Various meta-analyses have validated pretreatment NLR as a prognostic factor, with higher NLR values have been linked to poorer outcomes in numerous types of solid tumors [17-22]. This is true for PLR ratio as well, which has been found to be associated with somber outcomes considering OS and RFS [11].

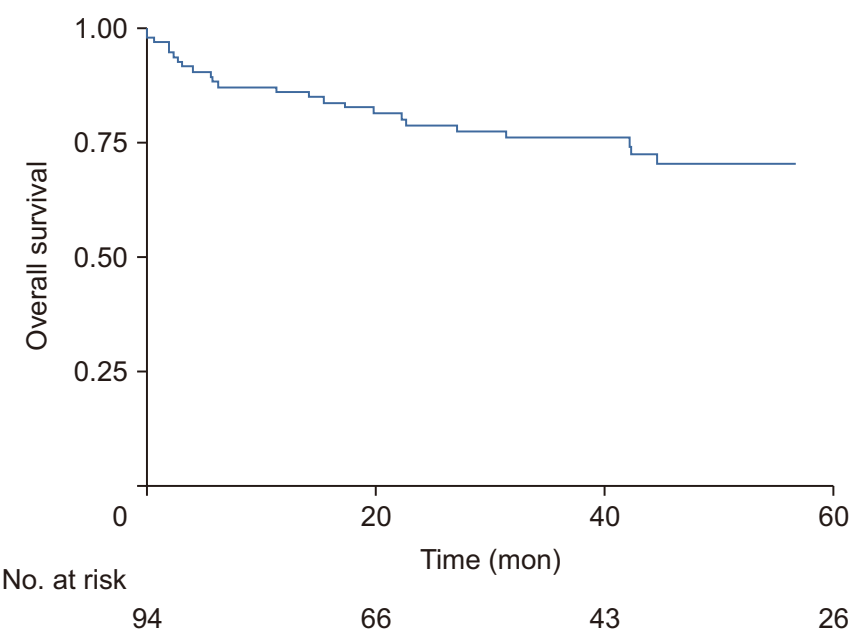

Fig. 3. The Kaplan-Meier curve showing overall survival of orthotopic liver transplantation patients. 
Table 3. Univariate and multivariate analyses for overall survival and recurrence-free survival in OLT patients

\begin{tabular}{|c|c|c|c|c|c|c|c|c|}
\hline \multirow{3}{*}{ Variable } & \multicolumn{4}{|c|}{ Overall survival } & \multirow{2}{*}{\multicolumn{4}{|c|}{ Recurrence-free survival }} \\
\hline & \multicolumn{2}{|c|}{ Univariate analysis } & \multicolumn{2}{|c|}{ Multivariate analysis } & & & & \\
\hline & $\mathrm{HR}(95 \% \mathrm{Cl})$ & $p$-value & $\mathrm{HR}(95 \% \mathrm{Cl})$ & $p$-value & $\mathrm{HR}(95 \% \mathrm{Cl})$ & $p$-value & $\mathrm{HR}(95 \% \mathrm{Cl})$ & $p$-value \\
\hline Age & $1.00(0.96-1.04)$ & 0.836 & & & $0.99(0.95-1.04)$ & 0.985 & & \\
\hline Male sex & $1.20(0.44-3.22)$ & 0.712 & & & $1.41(0.3-6.52)$ & 0.653 & & \\
\hline $\begin{array}{l}\text { Neoadjuvant } \\
\text { chemotherapy }\end{array}$ & $0.82(0.36-1.82)$ & 0.627 & & & $1.77(0.52-5.93)$ & 0.353 & & \\
\hline Single tumor & $1.67(0.74-3.77)$ & 0.213 & & & $1.69(0.53-5.38)$ & 0.372 & & \\
\hline Mayor nodule & $0.99(0.97-1.01)$ & 0.587 & & & $1.00(0.98-1.01)$ & 0.748 & & \\
\hline Total tumor size & $0.97(0.94-1.00)$ & 0.05 & $0.97(0.95-1.002)$ & 0.071 & $0.97(0.94-1.01)$ & 0.306 & & \\
\hline Cirrhosis & & & & & $0.47(0.05-3.78)$ & 0.478 & & \\
\hline Child Pugh A-B & $1.84(1.08-3.11)$ & 0.023 & $1.98(1.13-3.45)$ & 0.016 & $0.99(0.47-2.08)$ & 0.992 & & \\
\hline $\begin{array}{l}\text { Trending NLR } \\
\text { (late-baseline NLR) }\end{array}$ & $2.26(0.94-5.46)$ & 0.069 & & & $0.47(0.12-1.75)$ & 0.266 & & \\
\hline Trending PLR & $0.62(0.25-1.53)$ & 0.302 & & & $3.44(1.109-10.69)$ & 0.032 & $3.46(1.04-11.49)$ & 0.042 \\
\hline
\end{tabular}

OLT, orthotopic liver transplant; HR, hazard ratio; Cl, confidence interval; AFP, alpha-fetoprotein; NLR, neutrophil-to-lymphocyte ratio; PLR, platelet-tolymphocyte ratio.

However, few studies have investigated the correlation between these two markers and surgical outcomes of HCC patients undergoing OLT. NLR and PLR have mainly been analyzed as a static variable preceding a certain treatment [12]. However, inflammatory response is a dynamic continuum that is constantly affected not only by tumor and host biology, but also by treatment itself. This study aimed to analyze NLR and PLR as continuous variables measured before and after OLT to examine the prognostic value of their dynamic behavior by calculat-

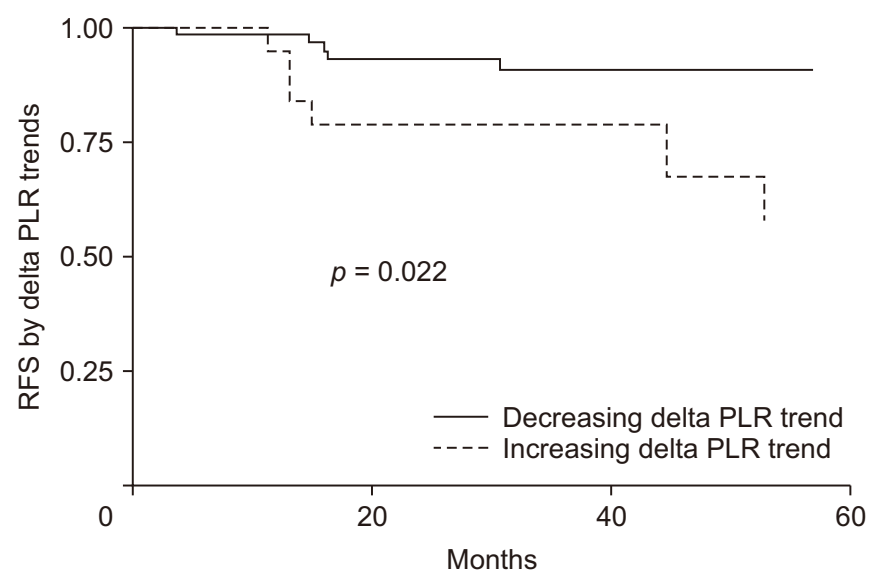

Fig. 4. The Kaplan-Meier curve showing recurrence-free survival (RFS) according to delta platelet-lymphocyte ratio (PLR) trends. ing delta NLR and delta PLR.

We analyzed multiples variables potentially related to OS and RFS using the Cox proportional hazards model. AFP > $100 \mathrm{ng} /$ $\mathrm{dL}$ was significantly associated with higher risk of recurrence $(p=0.026)$, in accordance with published literature stating that AFP could predict postoperative recurrence $[23,24]$. ChildPugh was also found to have a statistically significant relation with postoperative mortality $(p=0.016)$ in numerous studies regarding abdominal surgeries in cirrhotic patients $[25,26]$. An increasing delta NLR trend has been previously associated with poorer OS and RFS in patients with HCC undergoing curative resection [27]. To the best of our knowledge, no study has reported delta NLR/PLR in transplanted HCC patients. Our study did not show an association of delta NLR with OS or RFS. Delta PLR was found to have a statistically significant association with RFS $(p=0.042)$, but not OS $(p=0.302)$. An increasing trend in PLR might reflect a pro-inflammatory state that may lead to tumor proliferation and poor biological responses as previously described. It may even reflect an underlying undetected HCC in another part of the cirrhotic liver. Many studies have shown that inflammation can mediate tumor progression with a negative effect on patient survival [28].

The present study has some limitations. Its retrospective nature makes it susceptible to information bias. An attempt to reduce it was made by excluding patients who lacked adequate lab work and testing. Seven patients were excluded due to the absence of $\mathrm{CBC}$ on the month prior to surgery. Follow-up is 
also a problem with these patients, especially in our center. Being a referral center for transplantation for patients all over Argentina, many patients receive OLT in our institution but continue their follow-up in their hometowns or cities, leading to the loss of follow-up. Regardless, as a high-volume reference center, a homogeneous and large sample was obtained and analyzed. However, multivariate analyses taking potential confounders into account showed persistent worsening of RFS with delta PLR.

OLT is a potentially a life-saving therapy, with promising RFS and OS rates worldwide. Being able to predict early recurrence or mortality due to HCC in OLT is essential for patient selection and organ allocation, especially in countries in which donation is scarce with a great burden to the healthcare system. This study shows that inflammatory markers in OLT patients with HCC expressed as a dynamic value of "delta PLR" might be a useful, inexpensive, and simple tool to potentially predict recurrence outcomes. Benefits of utilizing delta NLR and delta PLR as prognostic markers mainly lie in the feasibility of their recollection are readily available in routine laboratory tests at minimal costs. A potential backside to these biomarkers is that their usefulness is limited to patients who have already undergone OLT. They could be used to exclude patients prior to treatment. Another possible limitation of this study was that post-OLT CBC might be influenced by immunosuppressive agents such as calcineurin inhibitors. This being said, it may serve as another factor in selecting patients for follow-up in a stricter fashion in order to detect early recurrence and treat it. Further potential studies regarding delta NLR/PLR are needed to validate them as useful biomarkers for OS and RFS in OLT for HCC and to determine possible cut-off values for each delta. In order to validate our findings, more large-scale research studies should be performed.

In conclusion, the analysis of PLR as a continuous variable may predict recurrence outcomes in patients with HCC undergoing OLT. It is more representative than individual values. This study shows that the dynamic behavior of PLR assessed through delta PLR is a useful tool that potentially allows prediction of recurrence outcomes in patients undergoing OLT.

\section{FUNDING}

The funding involved in this work has been provided by the National Cancer Institute of Argentina.

\section{CONFLICT OF INTEREST}

No potential conflict of interest relevant to this article was reported.

\section{ORCID}

Lucas José Caram, https://orcid.org/0000-0002-9312-5106

Francisco Calderon, https://orcid.org/0000-0003-2637-7909

Esteban Masino, https://orcid.org/0000-0002-5154-4660

Victoria Ardiles, https://orcid.org/0000-0002-4654-3970

Ezequiel Mauro, https://orcid.org/0000-0002-0757-7676

Leila Haddad, https://orcid.org/0000-0003-1332-5536

Juan Pekolj, https://orcid.org/0000-0003-2608-5914

Jimena Vicens, https://orcid.org/0000-0003-2978-7153

Adrian Gadano, https://orcid.org/0000-0002-3590-2472

Eduardo de Santibañes, https://orcid.org/0000-0002-5788-9694

Martín de Santibañes, https://orcid.org/0000-0002-0163-1248

\section{AUTHOR CONTRIBUTIONS}

Conceptualization: LJC, FC, E Masino, MS. Data curation: LJC, FC, E Masino, VA, E Mauro, LH, JV. Methodology: LJC, FC, E Masino, JP, AG, ES, MS. Visualization: LJC, FC, E Masino, VA, E Mauro, LH, AG. Writing - original draft: LJC, FC, E Masino, VA, MS. Writing - review \& editing: E Mauro, LH, JP, AG, ES.

\section{REFERENCES}

1. Bray F, Ferlay J, Soerjomataram I, Siegel RL, Torre LA, Jemal A. Global cancer statistics 2018: GLOBOCAN estimates of incidence and mortality worldwide for 36 cancers in 185 countries. CA Cancer J Clin 2018;68:394-424.

2. International Agency for Research on Cancer (IARC). Fact sheets by population-Globocan-IARC [Internet]. Lyon: IARC 2020 [cited 2016 Dec 18]. Available from: http://globocan.iarc.fr/Pages/fact_sheets_ population.aspx.

3. Heimbach JK, Kulik LM, Finn RS, Sirlin CB, Abecassis MM, Roberts LR, et al. AASLD guidelines for the treatment of hepatocellular carcinoma. Hepatology 2018;67:358-380.

4. Mazzaferro V, Regalia E, Doci R, Andreola S, Pulvirenti A, Bozzetti F, et al. Liver transplantation for the treatment of small hepatocellular carcinomas in patients with cirrhosis. N Engl J Med 1996;334:693699.

5. Yao FY, Ferrell L, Bass NM, Watson JJ, Bacchetti P, Venook A, et al. Liver transplantation for hepatocellular carcinoma: expansion of the tumor size limits does not adversely impact survival. Hepatology 2001;33:1394-1403.

6. Forner A, Reig M, Bruix J. Hepatocellular carcinoma. Lancet 2018;391:1301-1314.

7. Tandon P, Garcia-Tsao G. Prognostic indicators in hepatocellular carcinoma: a systematic review of 72 studies. Liver Int 2009;29:502-510.

8. Balkwill F, Mantovani A. Inflammation and cancer: back to Virchow? Lancet 2001;357:539-545.

9. Jackson JR, Seed MP, Kircher CH, Willoughby DA, Winkler JD. The codependence of angiogenesis and chronic inflammation. FASEB J 1997;11:457-465. 
10. Wang D, Bai N, Hu X, OuYang XW, Yao L, Tao Y, et al. Preoperative inflammatory markers of NLR and PLR as indicators of poor prognosis in resectable HCC. PeerJ 2019; 7:e7132.

11. Zhou X, Du Y, Huang Z, Xu J, Qiu T, Wang J, et al. Prognostic value of PLR in various cancers: a meta-analysis. PLoS One 2014;9:e101119.

12. Zheng J, Cai J, Li H, Zeng K, He L, Fu H, et al. Neutrophil to lymphocyte ratio and platelet to lymphocyte ratio as prognostic predictors for hepatocellular carcinoma patients with various treatments: a meta-analysis and systematic review. Cell Physiol Biochem 2017;44:967981.

13. Al Lawati Y, Cools-Lartigue J, Ramirez-GarciaLuna JL, Molina-Franjola JC, Pham D, Skothos E, et al. Dynamic alteration of neutrophil-to-lymphocyte ratio over treatment trajectory is associated with survival in esophageal adenocarcinoma. Ann Surg Oncol 2020;27:4413-4419.

14. Celik N, Kelly B, Soltys K, Squires JE, Vockley J, Shellmer DA, et al. Technique and outcome of domino liver transplantation from patients with maple syrup urine disease: expanding the donor pool for live donor liver transplantation. Clin Transplant 2019;33:e13721.

15. Tsochatzis EA, Germani G, Burroughs AK. Transarterial chemoembolization, transarterial chemotherapy, and intra-arterial chemotherapy for hepatocellular carcinoma treatment. Semin Oncol 2010;37:8993.

16. Guthrie GJ, Charles KA, Roxburgh CS, Horgan PG, McMillan DC, Clarke SJ. The systemic inflammation-based neutrophil-lymphocyte ratio: experience in patients with cancer. Crit Rev Oncol Hematol 2013;88:218-230.

17. Zhan H, Ma JY, Jian QC. Prognostic significance of pretreatment neutrophil-to-lymphocyte ratio in melanoma patients: a meta-analysis. Clin Chim Acta 2018;484:136-140.

18. Choi N, Kim JH, Chie EK, Gim J, Kang HC. A meta-analysis of the impact of neutrophil-to-lymphocyte ratio on treatment outcomes after radiotherapy for solid tumors. Medicine (Baltimore) 2019;98:e15369.
19. Yu Y, Wang H, Yan A, Wang H, Li X, Liu J, et al. Pretreatment neutrophil to lymphocyte ratio in determining the prognosis of head and neck cancer: a meta-analysis. BMC Cancer 2018;18:383.

20. Suh J, Jung JH, Jeong CW, Kwak C, Kim HH, Ku JH. Clinical significance of pre-treated neutrophil-lymphocyte ratio in the management of urothelial carcinoma: a systemic review and meta-analysis. Front Oncol 2019;9:1365.

21. Wang Z, Zhan P, Lv Y, Shen K, Wei Y, Liu H, et al. Prognostic role of pretreatment neutrophil-to-lymphocyte ratio in non-small cell lung cancer patients treated with systemic therapy: a meta-analysis. Transl Lung Cancer Res 2019;8:214-226.

22. Yuan D, Zhu K, Li K, Yan R, Jia Y, Dang C. The preoperative neutrophil-lymphocyte ratio predicts recurrence and survival among patients undergoing R0 resections of adenocarcinomas of the esophagogastric junction. J Surg Oncol 2014;110:333-340.

23. Duvoux C, Roudot-Thoraval F, Decaens T, Pessione F, Badran H, Piardi T, et al. Liver transplantation for hepatocellular carcinoma: a model including $a$-fetoprotein improves the performance of Milan criteria. Gastroenterology 2012;143:986-994.e3; quiz e14-e15.

24. Mazzaferro V, Sposito C, Zhou J, Pinna AD, De Carlis L, Fan J, et al. Metroticket 2.0 model for analysis of competing risks of death after liver transplantation for hepatocellular carcinoma. Gastroenterology 2018;154:128-139.

25. Mansour A, Watson W, Shayani V, Pickleman J. Abdominal operations in patients with cirrhosis: still a major surgical challenge. Surgery 1997;122:730-735; discussion 735-736.

26. Garrison RN, Cryer HM, Howard DA, Polk HC Jr. Clarification of risk factors for abdominal operations in patients with hepatic cirrhosis. Ann Surg 1984;199:648-655.

27. Peng W, Li C, Wen TF, Yan LN, Li B, Wang WT, Yang JY, et al. Neutrophil to lymphocyte ratio changes predict small hepatocellular carcinoma survival. J Surg Res 2014;192:402-408.

28. Liu J, Lin PC, Zhou BP. Inflammation fuels tumor progress and metastasis. Curr Pharm Des 2015;21:3032-3040. 